Mingorance Arnáiz, Cristina; Calvo Bernardino, Antonio (2013). Los resultados de los estudiantes en un procesó dēe evaluación con metodologías distintas. Revista de Investigación Educativa, 31 (1), 275-293.

http://dx.doi.org/10.6018/rie.31.1.153291

\title{
LOS RESULTADOS DE LOS ESTUDIANTES EN UN PROCESO DE EVALUACIÓN CON METODOLOGÍAS DISTINTAS
}

\author{
Cristina Mingorance Arnáiz, Antonio Calvo Bernardino \\ Universidad CEU San Pablo
}

\section{RESUMEN}

La utilización de una metodología docente basada en el fortalecimiento de distintas competencias exige la aplicación de un sistema de evaluación que permita valorar adecuadamente el logro de las competencias establecidas. Si bien, se suele decir que un sistema de enseñanza de este tipo perjudica el aprendizaje al reducir los conocimientos adquiridos. Nuestro objetivo es averiguar si la utilización de una metodología docente variada es positiva para la formación del alumno, así como si existe algún tipo de actividad que resulte más atractiva o más favorable para superar la asignatura. Para ello nos servimos de los resultados obtenidos por los alumnos de una misma asignatura a lo largo de varios cursos académicos.

Palabras clave: Formación en competencias; Sistemas de evaluación; Resultados de la evaluación.

\section{STUDENT RESULTS IN ASSESSMENT USING DIFFERENT METHODOLOGIES}

\section{ABSTRACT}

When we use a teaching methodology to strengthen different skills, we need to adapt our evaluation procedures to assess to what extend skills have been achieved. A teaching system

\footnotetext{
Correspondencia:

Ana Cristina Mingorance-Arnáiz: (mingor.fcee@ceu.es). Profesora Agregada de Economía Aplicada. Universidad CEU San Pablo. C/ Julián Romea no 23. Madrid 28003. Teléfono 91-456-63-36 (ext 5670).

Antonio Calvo-Bernardino: (acalvo@ceu.es). Catedrático de Economía Aplicada. Universidad CEU San Pablo. C/ Julián Romea no 23. Madrid 28003. Teléfono 91-456-63-36 (ext 5123).
} 
focused on teaching skills may have an adverse effect on learning. Our goal is to identify if the use of different teaching methodologies has a positive impact on student learning, and if there are particular kinds of tasks that may be more appealing or appropriate for student, so that they pass exams. In order to do this, we use results obtained by students during several academic years.

Keywords: Teaching skills, Evaluation procedures, Test marks.

\section{INTRODUCCIÓN}

El objetivo esencial de este trabajo es determinar el impacto que el empleo de una metodología de formación en competencias, que contemple diversos sistemas formativos y de evaluación, tiene sobre la formación del alumno. Para ello nos preguntamos sí los alumnos tienen preferencia por algún tipo de prueba concreta, y sí desarrollan más unas competencias que otras. También nos planteamos si la utilización de metodologías interactivas favorece la homogeneidad en la formación o la perjudica, así como sí, a medida que más signaturas han adoptado metodologías similares, los alumnos han modificado su patrón de trabajo resultándoles más sencillo el desarrollo de todas las competencias fijadas.

Para ello, hemos analizado los resultados obtenidos por los estudiantes en las pruebas realizadas en la asignatura de Sistema Financiero Español a lo largo de los cuatro últimos cursos académicos en los que venimos utilizando una metodología adaptada a Bolonia ${ }^{1}$.

Con el fin de conseguir una adecuada interpretación de los resultados, el artículo se divide, junto a esta introducción, en cuatro partes. En la segunda sección presentamos, brevemente, la metodología de análisis seguida en este trabajo, así como los principales interrogantes a los que pretendemos ofrecer una respuesta. En el tercer apartado presentamos la metodología docente empleada en la asignatura y los métodos de evaluación utilizados, haciendo referencia a las competencias, el sistema de evaluación, la distribución presencial y no presencial del esfuerzo del alumno, y al plan del curso. A continuación, en el siguiente apartado, analizaremos los resultados obtenidos por los alumnos, efectuando tanto un análisis horizontal, comparando los resultados de cada actividad formativa en los diferentes cursos académicos, como vertical, comparando los resultados en cada curso de las distintas actividades de evaluación realizadas. Todo ello se contempla teniendo en cuenta la valoración que el alumno hace de la metodología y el sistema de evaluación, así como de la dedicación y el nivel de aprendizaje. Terminamos el artículo destacando las principales conclusiones que se derivan de las calificaciones obtenidas por los alumnos en los distintos métodos empleados y en los diferentes cursos académicos, y respondiendo a los interrogantes inicialmente planteados.

1 Pese a que la asignatura se adaptó a Bolonia el curso 2006-2007, sólo se consideran los resultados de los últimos cuatro cursos académicos (de 2008-2009 a 2011-2012), cuando todas las asignaturas emplean una metodología Bolonia, y las competencias de la asignatura quedan definitivamente fijadas y articuladas en el conjunto del plan de estudios. 


\section{METODOLOGÍA DE INVETIGACIÓNY OBJETIVOS DEL TRABAJO}

La metodología de aprendizaje interactivo, que pretende el desarrollo de competencias, cuenta con un elevado número de defensores, quienes argumentan entre otras cosas, que favorece la formación integral del alumno, así como su capacidad de adaptación y auto-aprendizaje. Si bien, son también muchos los detractores de esta metodología de trabajo. Así, hay quien afirma que la formación en competencias reduce el aprendizaje conceptual y consecuentemente la preparación del alumno. Con este trabajo pretendemos conocer el impacto que la formación en competencias tiene en la formación del alumno y más en concreto si, una metodología de trabajo que busque el desarrollo de competencias, y no sólo de conocimientos, puede resultar insuficiente para la adecuada formación de nuestros universitarios.

Para dar respuesta a este interrogante nos hemos planteado algunas cuestiones parciales. En primer lugar, indagamos en las competencias generales y específicas que se desarrollan y evalúan con cada prueba realizada, y en segundo nos preguntamos sí los alumnos muestran preferencias por unas pruebas de evaluación frente a otras en función de las competencias evaluadas en cada una de ellas, lo que podría llevar al desarrollo adecuado de algunas, pero insuficiente de otras. Por otro lado, nos planteamos si una formación en competencias puede estar perjudicando la diversidad formativa entre alumnos o si la favorece. Nuestro último objetivo parcial, y dado que con el tiempo más asignaturas se han ido incorporando al aprendizaje interactivo, la formación en competencias y la evaluación continua, consiste en conocer si la metodología de trabajo se ha llegado a convertir en una rutina y los alumnos han adaptado su patrón de trabajo resultándoles más sencillo el desarrollo de las competencias fijadas.

Para responder a estos interrogantes hemos partido del análisis de los resultados académicos alcanzados por los alumnos de la asignatura de sistema financiero español de cuarto curso de las licenciaturas de Administración y Dirección de Empresas y de Economía, así como de la doble de Derecho y $\mathrm{ADE}^{2}$. Se han analizado los resultados de todas y cada una de las pruebas realizadas a lo largo de cuatro cursos académicos, al tiempo que se ha tenido en cuenta la valoración que los alumnos hacen de la metodología, su esfuerzo y el nivel de aprendizaje, para lo que nos hemos servido de una encuesta anónima que se realiza a todos los alumnos el último día de clase ${ }^{3}$. Dicha encuesta ha permanecido constante a lo largo de todos los cursos, y ha contado con un nivel de respuesta que se sitúa ligeramente por encima del $80 \%{ }^{4}$.

2 Sistema Financiero Español es una asignatura cuatrimestral, y obligatoria, que se imparte en las titulaciones de Economía y de Empresa con diferente número de créditos (5 ECTS en Empresa y 4 ECTS en Economía), lo que ha obligado a establecer algunas diferencias mínimas en el sistema de evaluación.

3 Para el análisis de los resultados académicos se ha acudido a las calificaciones obtenidas por los alumnos en las diferentes pruebas a lo largo de cuatro cursos, de 2008-2009 a 2011-2012. El número de alumnos matriculados, y por lo tanto evaluados, varía entre los 232 y los 245, si bien, algunos se han mantenido al margen del sistema de evaluación de competencias, ya sea por la no realización de las pruebas o por no cumplir con los requisitos mínimos de asistencia, por los que el número de alumnos considerados en el estudio varía entre los 167 del curso 2009-2010 y los 236 del curso 2011-2012. Por su parte, la participación en la encuesta que nos sirve para conocer la valoración del alumno, se sitúa entre las 162 respuestas en el curso 2009-2010 y los 218 el último curso considerado en el estudio.

4 Tanto el cuestionario que se realiza a los alumnos, como un análisis detallado de los resultados obtenidos se puede consultar en Calvo y Mingorance (2010 y 2011).

RIE, vol. 31-1 (2013) 


\section{METODOLOGÍAY COMPETENCIAS EVALUADAS}

Para poder comprender los resultados de los diferentes métodos de enseñanza, es necesario conocer previamente las metodologías docentes seguidas en la asignatura, las competencias que se pretenden desarrollar y el modo en que las mismas se evalúan.

Entendemos por competencia, y sin pretender ser exhaustivos en nuestra definición, "la combinación de conocimientos, habilidades, actitudes y valores que capacitan para afrontar con garantías la resolución de problemas o la intervención en un asunto, ya sea en un contexto académico, profesional o social" (Véase MEC (2006)) ${ }^{5}$. En este sentido, formar en competencias implica, como mencionan González López y López Cámara (2010), un cambio en la forma de pensar, sentir y actuar del estudiante, obligando a cambiar el enfoque educativo que debe orientarse a quién aprende y a las demandas crecientes de la sociedad.

En cuanto a las competencias a alcanzar, éstas se han ido adaptando poco a poco, como se propone en Fernández (2006), a las exigencias del EEES, favoreciendo cada vez más la participación de los estudiantes en el proceso de enseñanza-aprendizaje. En este sentido, y como se señala en Calvo y Mingorance (2010), las competencias que complementan los conocimientos teóricos fueron fijadas con el consenso de todos los profesores de la asignatura, y se han adaptado parcialmente, tratando de progresar en las competencias alcanzadas por los estudiantes en otras asignaturas previas, a medida que la metodología Bolonia se ha impuesto en los diferentes cursos. Se eliminaron algunas competencias y se incorporaron otras, al tiempo que se modificó el grado de consecución de algunas de las que se mantuvieron.

Entre las competencias generales, cabe destacar la capacidad de organización y planificación, el desarrollo de la responsabilidad y de la madurez personal y académica, la honestidad, el desarrollo de un cierto grado de independencia en su proceso de aprendizaje, el trabajo en equipo y el progreso en las relaciones interpersonales. Por su parte, entre las competencias específicas que complementan los conocimientos propios de la asignatura encontramos, la capacidad para analizar la situación financiera del país, así como para interpretar el funcionamiento de las instituciones y de los mercados financieros de nuestra economía, siendo capaz de juzgar su utilidad en el crecimiento económico. Del mismo modo, el alumno deberá conocer las características de los activos financieros y tomar decisiones sobre las mejores inversiones económicas. El manejo de bases de datos específicas de carácter financiero, la gestión de la información obtenida, y la capacidad para expresar de forma coherente, ordenada y razonada sus opiniones y valoraciones sobre la situación financiera del país, son considerados asimismo elementos esenciales sobre los que se asienta la estructura metodológica y de evaluación de la asignatura.

5 Para un análisis más completo del concepto de competencia puede consultarse Rodríguez Esteban y Vierira Aller (2009), donde se ofrecen diferentes definiciones de competencia, así como una interpretación de lo que la formación en competencias implica. 


\section{I. La metodología de enseñanza y aprendizaje empleada}

Una vez definida la asignatura y sus competencias se estableció la metodología docente a seguir para lograrlas, así como los criterios de evaluación que permitiesen valorar su grado de consecución.

En relación con la metodología, la asignatura se divide en tres bloques, todos ellos, como se recoge en la figura 1, con la misma estructura, constando de una lección magistral, una serie de seminarios, y un taller con su correspondiente tutoría conjunta.

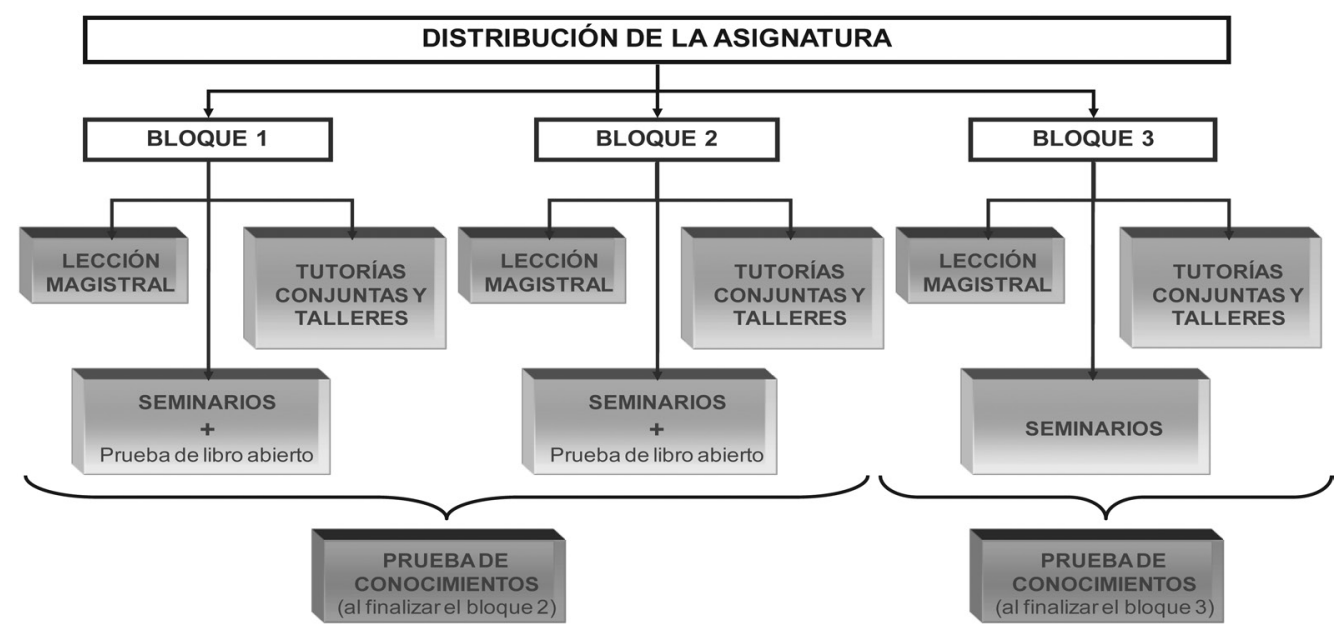

FIGURA 1

ESTRUCTURA METODOLÓGICA DE LA ASIGNATURA

Fuente: Elaboración propia

La lección magistral, con la que se abre cada bloque, es impartida por el Catedrático y responsable de la Unidad Docente. Es común para todos los alumnos matriculados en la asignatura, y ofrece una visión general del bloque, situando al alumno en el contexto de los temas que se explican en el mismo. Para garantizar su aprovechamiento, y cumplir con algunas de las competencias fijadas, al cierre de la sesión se realiza una prueba test sobre el contenido tratado en la lección expositiva.

Los seminarios guardan una relación estrecha con las clases tradicionales, y en ellos, cada profesor, con su grupo de clase, cubre los conocimientos teóricos que debe adquirir el alumno, profundizando en los aspectos más relevantes del aprendizaje de una manera dinámica y reflexiva. El profesor facilita al alumno documentación básica (libro de texto, transparencias y artículos) que le sirven para preparar las clases con antelación, lo que favorece la participación del alumno mediante preguntas que dinamizan la clase beneficiando el aprendizaje ${ }^{6}$.

6 Al facilitarle cierta documentación se espera que el alumno la lea con carácter previo, lo que permite que el profesor que imparte el seminario pueda centrarse en los aspectos esenciales que requieren una mayor profundización. 
En los talleres, que sirven de cierre al bloque, se desarrollan los aspectos más prácticos de la asignatura. Cada taller, de cuatro horas de duración, se compone de dos partes: una primera que es la tutoría, realizada entre 10 y 15 días antes de la segunda parte que es el taller. En la tutoría se explican los conceptos teóricos necesarios para la realización de cada taller, al tiempo que se dan las instrucciones necesarias para la realización del mismo. Por su parte, en el taller, los alumnos presentan el trabajo conjunto $^{7}$, realizado fuera del aula, bajo las directrices fijadas por el profesor, pudiendo diferir éstas de unos profesores a otros y de unos talleres a otros en función de las competencias que se pretendan potenciar en cada uno ${ }^{8}$.

Las tutorías conjuntas y talleres merecen, por su dinámica y diseño, una mención especial, pues si bien no constituyen actualmente una novedad en la universidad, sí que se organizan atendiendo a unos criterios y unos objetivos que son esenciales para la consecución de las competencias fijadas al principio del curso. En este sentido, cada profesor de la Unidad Docente ofrece, para cada uno de los bloques en los que se divide la asignatura, un tema de taller con tres horarios distintos, siendo la oferta total de doce talleres alternativos de los que los alumnos deben elegir exclusivamente uno ${ }^{9}$. Esta posibilidad de elección le permite desarrollar su madurez, pues se espera que la elección se realice en función del interés por el tema tratado en cada uno de los talleres. Además, y en la medida en que en ellos se promueve el debate y se anima a los alumnos a defender sus posiciones, siempre respetando y valorando el trabajo de los demás, se les ayuda a desarrollar su capacidad de relación interpersonal, así como a defender sus posiciones, resolver problemas y gestionar la información, entre otras competencias. Mientras la lección magistral es única para todos los alumnos matriculados en la asignatura, y los seminarios, con un mismo contenido (el material docente facilitado a los alumnos para su seguimiento es el mismo para todos los profesores), pueden diferir de unos grupos a otros en función del profesor que los imparte; los talleres difieren, tanto en contenido como en sistema de evaluación, y son los alumnos quienes libremente deciden, en función de sus intereses personales y formativos, el taller que desean realizar en cada bloque ${ }^{10}$.

\subsection{El sistema de evaluación}

Definida la metodología, es necesario, como se menciona en Yáñiz (2006), diseñar el sistema de evaluación que se empleará en la valoración de los estudiantes, y realizar la

7 La mayoría de los trabajos en esta actividad se desarrollan en grupos. En este sentido, son muchos los trabajos que destacan el impacto de la actuación en grupo para la formación en competencias del alumnado, así como la mejor forma de llevar a cabo la evaluación. Véase, por ejemplo, Moust et al. (2007).

8 Las diferencias en las competencias desarrolladas y evaluadas en los talleres impartidos por los distintos profesores son fundamentalmente de matiz e intensidad, pues el objetivo de la Unidad Docente, y así se establece al principio del curso, es formar al alumno en unos conocimientos y competencias mínimas que en cualquier caso deben ser garantizadas.

9 El horario de la tutoría es único para todos los alumnos que deciden matricularse del taller con un profesor, mientras que para favorecer la participación, y mejorar el seguimiento y evaluación del alumno, éstos se dividen en tres grupos de no más de 25 alumnos a la hora de poner en común el trabajo desarrollado.

10 Aunque el contenido y el sistema de evaluación difiera de unos talleres a otros, a la hora de diseñarlo los profesores tienen presente las competencias fijadas por la unidad docente. 
planificación del curso. Ambos se dan a conocer el primer día de clase con el objetivo de que los alumnos puedan responsabilizarse de su propio trabajo. La planificación se ajusta, por un lado, a las horas de clase efectivas con las que se cuenta (descontados los festivos), y por otro, a la estructura de clases diseñada, buscando una distribución equitativa entre la carga docente asignada a cada actividad metodológica y el número de horas dedicadas presencialmente a su desarrollo.

Para lograr que los alumnos alcancen las competencias definidas, no es suficiente con una estructura metodológica como la descrita, y se hace necesario el diseño de un sistema de evaluación continuo, variado y formativo (Inda et al. (2008) y Segura (2009)). Así, en línea con Cano (2008), se evalúan todas las metodologías con actividades que permiten valorar tanto la adquisición de conocimientos como de habilidades y actitudes. En concreto, las lecciones magistrales se califican con pruebas tipo test, de carácter general, realizadas al final de las mismas, y que resultan esenciales para evaluar, entre otros aspectos, la capacidad para analizar y sintetizar, pero sobre todo para gestionar la información financiera facilitada por el docente.

Los seminarios y los talleres son las metodologías más completas, y también los que nos permiten evaluar un mayor número de competencias. Las competencias de aprendizaje autónomo, análisis y síntesis de la información, así como la madurez del alumno, se califican mediante dos pruebas a libro abierto sobre aspectos específicos no tratados en el aula. Los resultados de dichas pruebas, pese a formar parte de los seminarios, se consideran junto a los de las lecciones magistrales. A su vez, los seminarios cuentan con dos pruebas parciales liberatorias con preguntas de desarrollo y un test general al final del curso con las que se busca comprobar la adquisición, no sólo de conocimientos, sino también, la capacidad de formular argumentos teóricos y aplicados sobre el sistema financiero de una manera razonada, de sintetizar los conocimientos alcanzados, de comunicar adecuadamente ideas económico-financieras, y de resolver las cuestiones y problemas planteados..

Por su parte, en la valoración de las tutorías conjuntas y los talleres, hay una mayor variación; pues si bien todos los profesores realizan una prueba test al final de la tutoría conjunta, el modo en que se evalúa el trabajo realizado por el alumno en el taller cambia de unos talleres a otros. No obstante, el modo en que éstos se desarrollan y evalúan debe permitir el cumplimiento de los objetivos fijados en la guía docente, y la formación en todas las competencias. Para ello, los miembros de la Unidad Docente establecen reuniones donde se pone en común el contenido concreto del taller (cada profesor fija su propio tema evitando solapamientos, lo que permite una formación específica y particular del alumno), el modo en que se desarrollará el mismo, el trabajo que deberán realizar los alumnos y la forma en que se evaluarán las competencias a desarrollar ${ }^{11}$. En todos los talleres se valora el trabajo en equipo, la comunicación escrita y oral, la capacidad de análisis y síntesis, la de razonamiento crítico y la toma de decisiones y resolución de problemas y conflictos, así como el manejo de bases de datos. También se valora el desarrollo de las relaciones interpersonales y de la justicia,

11 De este modo se garantiza que, con independencia de los talleres elegidos por los alumnos, todos ellos adquieran una formación mínima en las competencias, tanto generales como específicas, que se pretenden desarrollar. 
pues el modo en que se diseñan las exposiciones hace que el resultado de cada uno de los miembros del grupo dependa del trabajo de todos sus integrantes.

TABLA 1

COMPETENCIAS ALCANZADAS CON LAS PRUEBAS DE EVALLUACIÓN CONTINUA DISEÑADAS

\begin{tabular}{|c|c|c|c|c|c|c|c|}
\hline & $\begin{array}{l}\text { Prueba } \\
\text { parcial }\end{array}$ & $\begin{array}{c}\text { Libro } \\
\text { abierto }\end{array}$ & Magistral & $\begin{array}{c}\text { Tutoría } \\
\text { conjunta }\end{array}$ & Taller & Metodología & $\begin{array}{c}\text { Actitud } \\
\text { U.D. }\end{array}$ \\
\hline \multicolumn{8}{|l|}{ Aprendizaje autónomo } \\
\hline \multicolumn{8}{|l|}{ Mejora del trabajo en equipo } \\
\hline \multicolumn{8}{|l|}{ Relaciones interpersonales } \\
\hline \multicolumn{8}{|l|}{ Análisis y síntesis } \\
\hline \multicolumn{8}{|l|}{ Gestión información } \\
\hline \multicolumn{8}{|l|}{ Adaptación nuevas situaciones } \\
\hline \multicolumn{8}{|l|}{ Comunicación escrita } \\
\hline \multicolumn{8}{|l|}{ Razonamiento crítico } \\
\hline \multicolumn{8}{|l|}{ Toma de decisiones } \\
\hline \multicolumn{8}{|l|}{ Comunicación oral } \\
\hline \multicolumn{8}{|l|}{ Organización y planificación } \\
\hline \multicolumn{8}{|l|}{ Trabajar en entornos de presión } \\
\hline \multicolumn{8}{|l|}{ Defensa de tareas asignadas } \\
\hline \multicolumn{8}{|l|}{ Compromiso ético } \\
\hline \multicolumn{8}{|l|}{ Madurez y responsabilidad } \\
\hline \multicolumn{8}{|l|}{ Honestidad } \\
\hline \multicolumn{8}{|l|}{ Justicia } \\
\hline \multicolumn{8}{|l|}{ Conocimientos } \\
\hline \multicolumn{8}{|l|}{ Resolución de problemas } \\
\hline Manejo de bases de datos financieras & & & & & & & \\
\hline
\end{tabular}

Competencia fundamental de la actividad

Competencia secundaria de la actividad

Fuente: Elaboración propia a partir de la Guía Docente de Sistema Financiero Español (Curso 2011-2012)

En resumen, como se deduce de la tabla 1, ni todas las pruebas pretenden evaluar todas las competencias, ni todas las competencias se pueden cubrir con todas las pruebas realizadas. Además, la intensidad con la que se evalúan las competencias puede variar de una prueba a otra, y el modo en que se hace también.

Algunos valores y actitudes como el compromiso ético, la madurez y la honestidad, tratan de inculcarse al alumno, mediante la propia actitud de trabajo y respeto que los miembros de la Unidad Docente muestran entre ellos y para con los alumnos, tanto dentro como fuera del aula, y por otro, mediante la realización de los talleres, cuyo sistema de evaluación hace que la actitud de unos alumnos influya en la nota final de $\operatorname{otros}^{12}$.

12 La necesidad de crear grupos docentes y trabajar conjuntamente en las Unidades Docentes se ha puesto de manifiesto en muchos trabajos, entre ellos el de Ion y Cano (2011). 
Una vez establecidas las pruebas con las que evaluar la adquisición de competencias, hay que asignar las ponderaciones de cada una en la calificación final del alumno, lo que se realiza teniendo en cuenta, el tiempo dedicado a su desarrollo, y las competencias que dicha actividad permite alcanzar y por lo tanto evaluar ${ }^{13}$.

Las lecciones magistrales y las pruebas a libro abierto tienen una ponderación en la calificación final del 10\% en ambas titulaciones. Por su parte, las dos pruebas parciales de desarrollo (80\%) y el test final de conocimientos generales (20\%) que cubre todo el temario, tienen un peso en la calificación final del 50\% en ADE y del 40\% en Economía debido al menor número de horas presenciales que se dedica a seminarios en la última ${ }^{14}$. Finalmente, para calificar las tutorías conjuntas y los talleres se realiza una prueba test al cierre de la tutoría cuya puntuación es el $20 \%$ de la nota del taller, mientras el $80 \%$ restante es el resultado del trabajo que debe preparar el alumno de cara al taller, siendo el profesor que lo imparte el que lo distribuye según sus propios criterios $^{15}$.

\subsection{Distribución del esfuerzo del alumno y plan del curso}

Dada la estructura metodológica y el sistema de evaluación, se realiza la planificación del curso, que permite a profesores y alumnos organizar y planificar su propio trabajo. La planificación se ajusta, por un lado, a las horas de clase efectivas con las que se cuenta, y por otro, a la estructura de clases diseñada, buscando una distribución equitativa entre la carga docente asignada a cada actividad y el número de horas dedicadas a su desarrollo.

Antes de establecer el plan del curso, y al mismo tiempo que se está definiendo el sistema de evaluación, la Unidad Docente, en línea con los ECTS con los que cuenta la asignatura en cada una de las titulaciones, distribuye las horas de esfuerzo total entre presencial y autónomo ${ }^{16}$. En concreto, los profesores distribuyen el esfuerzo presencial y no presencial necesario para que un alumno esté en disposición de superar la asignatura (tabla 2).

Una vez distribuido el esfuerzo presencial y no presencial, se elabora el plan del curso, haciéndose el reparto semanal de la carga de trabajo con el fin de evitar la sobrecarga de trabajo y facilitar el aprendizaje continuo del alumno a lo largo del semestre.

13 En línea con lo indicado por Muñiz y Fonseca (2008), el establecimiento de estas pruebas se ha realizado en base a un proceso paulatino de aprendizaje de los que componen la unidad docente, ya que los profesores universitarios hemos llegado a este cambio metodológico con una escasa formación en estas prácticas, como también señalan Brennan (2006) y Joint Committee on Standards for Educational Evaluation (2003).

14 Para evitar que los alumnos que no alcanzan la nota mínima en las pruebas escritas de conocimiento abandonen la asignatura, se les realiza, junto al test final, una prueba de la parte no liberada, pero para no romper con la idea de la evaluación continua, y procurar un esfuerzo permanente de los alumnos, la nota obtenida en la prueba parcial hace media con la que obtuvieron en la prueba que repiten.

15 El peso de los talleres en la calificación final del alumno varía en función de las titulaciones, siendo mayor la ponderación para los alumnos de Economía (50\%) que para los de ADE (40\%).

16 La forma en que se distribuye este esfuerzo se desarrolla en muchos trabajos. Véase, por ejemplo, Calvo y Mingorance (2011) y la bibliografía allí citada. 
TABLA 2

DISTRIBUCIÓN DEL ESFUERZO DEL ALUMNO POR BLOQUES EN CADA UNA DE LAS TITULACIONES PARA EL CURSO 2011-2012

\begin{tabular}{lcccccccc}
\hline & \multicolumn{2}{c}{ Bloque 1} & \multicolumn{2}{c}{ Bloque 2} & \multicolumn{2}{c}{ Bloque 3} & \multicolumn{3}{c}{ Total } \\
& ADE & Economía & ADE & Economía & ADE & Economía & ADE & Economía \\
\cline { 2 - 10 } Trabajo presencial & $\mathbf{1 5}$ & $\mathbf{1 4}$ & $\mathbf{1 3}$ & $\mathbf{1 2}$ & $\mathbf{2 8}$ & $\mathbf{2 2}$ & $\mathbf{5 6}$ & $\mathbf{5 0}$ \\
$\quad$ Presentación & 2 & 2 & & & & & 2 & 2 \\
$\quad$ Lección magistral & 2 & 2 & 2 & 2 & 2 & 2 & 6 & 6 \\
Seminario (*) & 6 & 5 & 6 & 5 & 18 & 14 & 30 & 24 \\
Tutoría conjunta & 2 & 2 & 2 & 2 & 2 & 2 & 6 & 6 \\
Taller & 2 & 2 & 2 & 2 & 2 & 2 & 6 & 6 \\
Prueba parcial & 1 & 1 & 1 & 1 & 2 & 2 & 4 & 4 \\
$\quad$ Prueba Final & & & & & 2 & 2 & 2 & 2 \\
Trabajo autónomo & $\mathbf{2 5}$ & $\mathbf{1 8}$ & $\mathbf{2 6}$ & $\mathbf{2 0}$ & $\mathbf{4 7}$ & $\mathbf{3 4}$ & $\mathbf{9 4}$ & $\mathbf{7 0}$ \\
$\quad$ Lección magistral & & & & & & & & \\
Seminario $(*)$ & 18 & 12 & 19 & 14 & 39 & 28 & 76 & 52 \\
$\quad$ Pruebas parciales & 10 & 6 & 10 & 7 & 21 & 16 & 41 & 29 \\
$\quad$ Prueba final & 8 & 5 & 9 & 6 & 18 & 12 & 35 & 23 \\
$\quad$ Taller & 6 & 6 & 6 & 6 & 6 & 6 & 18 & 18 \\
\hline Horas totales de esfuerzo & $\mathbf{4 0}$ & $\mathbf{3 2}$ & $\mathbf{3 9}$ & $\mathbf{3 2}$ & $\mathbf{7 1}$ & $\mathbf{5 6}$ & $\mathbf{1 5 0}$ & $\mathbf{1 2 0}$ \\
\hline
\end{tabular}

Fuente: Elaboración propia a partir de la Guía Docente de Sistema Financiero Español (Curso 2011-2012)

$\left(^{*}\right)$ Los seminarios incluyen el tiempo dedicado a las pruebas a libro abierto.

\section{PRINCIPALES RESULTADOS}

Descrita la metodología docente, las competencias a desarrollar y las pruebas vinculadas a cada proceso de enseñanza, vamos a detenernos en el análisis de los resultados que, para cada actividad de evaluación, se obtienen cada curso académico. Compararemos las calificaciones de las diferentes pruebas dentro de un mismo curso, así como las de una misma prueba en los distintos cursos tratando de responder a los interrogantes inicialmente planteados. Y, basándonos en la encuesta planteada, consideraremos la valoración de la metodología de trabajo y de su aprendizaje para cada una de las actividades efectuada el alumno.

Antes de ello, conviene precisar algunas cuestiones. En primer lugar, tanto los resultados académicos como los de la encuesta abarcan cuatro cursos académicos en los que la estabilidad de los miembros de la unidad docente y de la metodología empleada es grande, por eso, y aunque de acuerdo con Andreu-Andrés y Labrador-Piquer (2011) cabe esperar que los resultados se afinen con el tiempo, consideramos que los mismos son suficientemente significativos.

En segundo, debemos mencionar que a lo largo de todos los cursos considerados los criterios de formación y evaluación han sido similares, dándose la paradoja de que alguna prueba ha sido común e idéntica todos los años. En concreto, la prueba de las lecciones magistrales, el test final ligado a los seminarios, y en algunos casos las pruebas de libro abierto. Para evitar que las preguntas se filtrasen de un curso a otro las pruebas consistieron en preguntas test con cuatro posibles respuestas. Además, en 
ocasiones las mismas se proyectaban sobre la pantalla del aula una a una, y por un período corto de tiempo.

En el resto de actividades, realización y presentación de trabajos en los talleres, y preguntas de desarrollo en las pruebas vinculadas a las clases tradicionales, se han introducido modificaciones a lo largo de los cursos para evitar que las preguntas y los trabajos se filtrasen. No obstante, el carácter y estructura de los mismos, así como las competencias evaluadas han sido muy parecidas todos los años.

Por tanto, los resultados finales que vamos a ofrecer van a depender, en nuestra opinión, de dos tipos de factores: por un lado, de la calidad y del esfuerzo del alumnado en cada curso académico; y, por otro, de la mayor o menor adaptación que hayan tenido a esta metodología a medida que han ido pasando los años ${ }^{17}$.

\section{I. Satisfacción general del alumno y resultados medios de las pruebas}

En general, el alumno afirma sentirse satisfecho tanto con la metodología seguida, como con el modo en que se distribuyen las calificaciones entre las distintas pruebas (véase tabla 3).

TABLA 3

NIVEL DE SATISFACCIÓN DEL ALUMNO

\begin{tabular}{|c|c|c|c|c|c|c|c|c|}
\hline & \multicolumn{4}{|c|}{ Con la metodología } & \multicolumn{4}{|c|}{ Con la distribución de las calificaciones } \\
\hline & $2008-2009$ & $2009-2010$ & $2010-2011$ & 2011-2012 & 2008-2009 & $2009-2010$ & 2010-2011 & 2011-2012 \\
\hline Muy satisfecho & $8,2 \%$ & $12,4 \%$ & $9,8 \%$ & $15,5 \%$ & $10,6 \%$ & $6,8 \%$ & $4,3 \%$ & $15,1 \%$ \\
\hline Satisfecho & $63,7 \%$ & $61,1 \%$ & $54,0 \%$ & $60,3 \%$ & $63,9 \%$ & $60,3 \%$ & $50,0 \%$ & $58,9 \%$ \\
\hline Poco satisfecho & $8,8 \%$ & $11,7 \%$ & $10,4 \%$ & $11,4 \%$ & $8,9 \%$ & $12,4 \%$ & $11,1 \%$ & $12,8 \%$ \\
\hline Totalmente insatisfecho & $19,2 \%$ & $14,8 \%$ & $25,8 \%$ & $12,8 \%$ & $16,7 \%$ & $20,5 \%$ & $34,6 \%$ & $13,2 \%$ \\
\hline
\end{tabular}

Fuente: Elaboración propia.

Pese a esta satisfacción inicial, los resultados, como comprobamos en la tabla 4, son en general menos satisfactorios. La actividad en la que los alumnos han obtenido una calificación media mayor, ha sido la de las pruebas magistrales, con un 6,53, siendo la diferencia entre las propias pruebas vinculadas a las lecciones magistrales $(6,49)$ y las que se desarrollan a libro abierto $(6,54)$ de apenas 5 centésimas.

17 Somos conscientes de que existen otros factores que pueden influir en los resultados de los alumnos en cada actividad y en cada curso académico. Así, cabe destacar, en primer lugar, el propio estado de ánimo del profesorado al corregir las pruebas de desarrollo o el trabajo de los talleres, pues existe siempre un cierto componente de subjetividad que se contrarresta en parte, como hemos indicado, en que han sido los mismos profesores los que han impartido la materia en los cursos contemplados en el análisis. En segundo lugar, la presencia de pruebas de otras asignaturas en períodos próximos a la realización de alguna de las de nuestra materia, lo que condiciona sin duda el tiempo dedicado al trabajo no presencial. En tercero, la explicación del profesor si de ella los alumnos tienen que responder, de forma inmediata, a diversas preguntas; etc. Son todos ellos, en cualquier caso, factores de difícil internalización, y que condicionan los resultados comparativos de cualquier asignatura, por lo que nosotros los vamos a considerar exógenos, y que, por tanto, su influencia, o no se produce, o es similar para todas las actividades y todos los cursos académicos. 
TABLA 4

VALORES MEDIOS TOTALES Y DESVIACIONES DE CADA CURSO RESPECTO AL TOTAL

\begin{tabular}{lccccc}
\hline & $2008-2012$ & $2008-2009$ & $2009-2010$ & $2010-2011$ & $2011-2012$ \\
\cline { 2 - 6 } Magistrales & 6,53 & 0,08 & $-0,22$ & 0,10 & $-0,04$ \\
Lecciones Magistrales & 6,49 & 0,11 & $-0,43$ & 0,27 & $-0,02$ \\
Libro Abierto & 6,54 & 0,09 & $-0,01$ & $-0,05$ & $-0,02$ \\
Talleres & 6,35 & 0,13 & 0,45 & $-0,37$ & $-0,13$ \\
Seminarios & 4,36 & 0,47 & $-0,13$ & $-0,31$ & 0,00 \\
Bloques de teoria & 4,10 & 0,34 & $-0,01$ & $-0,37$ & 0,05 \\
Test & 5,41 & 0,96 & $-0,59$ & $-0,05$ & $-0,22$ \\
\hline Nota final & $\mathbf{5 , 4 3}$ & $\mathbf{0 , 2 7}$ & $\mathbf{0 , 1 3}$ & $\mathbf{- 0 , 2 9}$ & $\mathbf{- 0 , 0 6}$ \\
\hline
\end{tabular}

Fuente: Elaboración propia.

En la misma línea se sitúan los resultados de los talleres, con una calificación media para los cuatro cursos de 6,35. Muy lejos de esta puntuación se sitúan los seminarios, donde la calificación media es de suspenso $(4,36)$ debido a las preguntas de desarrollo $(4,10)$, siendo la nota media del test final de 5,41 , muy próxima a la nota media final obtenida por los alumnos en la asignatura $(5,43)$.

Así, puede decirse que bajo una metodología como la descrita, el aprendizaje del alumno, aunque suficiente para aprobar, tiene unas marcadas preferencias por las actividades no memorísticas, obteniéndose los peores resultados en los bloques teóricos ${ }^{18}$. En general, el alumno parece preferir actividades prácticas o de resolución rápida como los test, frente a aquellas que requieren una mayor dedicación en horas de estudio. De hecho, afirman dedicar más tiempo a la realización de los talleres que a la preparación de las pruebas de los seminarios, al menos en comparación con el tiempo que la Unidad Docente considera que debe dedicarse a cada actividad (Calvo y Mingorance (2011)).

En este sentido, podemos señalar que el alumno se decanta claramente por unas competencias frente a otras, destacando la capacidad de trabajado en grupo, el desarrollo de las relaciones interpersonales, la gestión de la información, la toma de decisiones y la comunicación oral. Este hecho puede deberse a que le exige un menor esfuerzo en horas de preparación, pero también a que el alumno considera que el desarrollo de estas competencias le prepara mejor para el mercado laboral.

Por otro lado, cabe señalar que existen discrepancias entre los resultados obtenidos en los diferentes cursos. Los mejores resultados medios se logran en los dos primeros cursos académicos, mientras que en 2010-2011, y ligeramente en el último de los cursos considerados, la calificación media obtenida es inferior a la del período completo. Este hecho pone de manifiesto un empeoramiento de los resultados globales.

Además, la desviación entre la nota media obtenida en cada curso y la nota media del total de los cursos académicos considerados, para cada una de las pruebas, es siempre positiva en el curso 2008-2009, mientras que se torna en negativa para todas las pruebas, con la única excepción de los talleres, el siguiente curso. Por bloques,

18 Resulta llamativo que la satisfacción del alumno no sólo con la metodología, sino también con el nivel de aprendizaje alcanzado, sea elevado. Así, más del 75\% de los alumnos, y con independencia del curso académico, dice sentirse satisfecho con el aprendizaje. 
las mejores calificaciones para la parte de magistrales se alcanzan el curso 2010-2011. En el caso de los talleres, destaca especialmente el curso 2009-2010 con una nota media de 6,80. Finalmente, y en la parte de los seminarios debe señalarse que sólo en el primero de los cursos considerados, 2008-2009, la nota media logra acercarse al aprobado $(4,83)$, así como que es en el bloque del test final donde los resultados son mejores, siendo la nota media de suspenso sólo en el curso 2009-2010. En este sentido, y pese a los peores resultados que se han ido obteniendo a medida que en otras asignaturas se han utilizado metodologías similares, no podemos afirmar que exista un claro patrón de comportamiento en los alumnos. Sin embargo, sí parece que el alumno reduce su dedicación a la asignatura cuando en otras se emplean métodos similares.

\subsection{Modificación del aprendizaje por cursos académicos}

Llegados a este punto debemos profundizar en el análisis detallado de las calificaciones obtenidas en cada actividad y cada curso académico considerado. En general, la calificación más repetida ha sido el aprobado, e incluso el notable. Sólo en los seminarios, y en el tercer curso académico en el caso de los test, podemos decir que la nota más repetida ha sido el suspenso. Si bien, la calificación central (mediana) es siempre de aprobado con la única excepción de los seminarios, y el del test final en el segundo curso.

Para profundizar en los resultados obtenidos por los alumnos en las diferentes pruebas de evaluación presentamos la distribución de suspensos, aprobados, notables y sobresalientes, por actividad y curso académico. Hemos establecido una diferencia en el grupo de suspensos entre aquellos cuya calificación es superior al 3,5 y por tanto tienen opción a aprobar, y aquellos que por ser su calificación inferior al 3,5 no pueden aprobar en la convocatoria ordinaria.

Del contenido de la tabla 5 se puede destacar, en primer lugar, que es en las pruebas de desarrollo, correspondientes al bloque de seminarios, en las que el porcentaje de suspensos, independientemente del curso académico, es más elevado, superándose siempre el 60\%. Además, y salvo en el curso académico 2010-2011 donde el número de los que no libera es muy superior, la distribución entre los que tienen acceso a liberar y los que no es prácticamente igual.

En segundo lugar, y como ya hemos adelantado, parece que los alumnos se adaptan mejor a la prueba tipo test, donde la necesidad de memorizar es menor, y donde el factor suerte puede desempeñar un cierto papel. No obstante, se observa un empeoramiento notable en los resultados del test, y mientras el primer curso académico sólo el 18\% suspendía, este porcentaje se eleva al $40 \%$ o $50 \%$ el resto de años.

Por su parte, las calificaciones de los talleres, seguidas de las de las pruebas a libro abierto, son sin duda las más elevadas, no sólo por el número total de alumnos que obtienen una nota de aprobado o superior (prácticamente por encima del 80\%), sino porque en ellos el número de los que alcanza el notable es importante. Así, en el caso de las segundas, el porcentaje se sitúa alrededor del 35\% todos los años, al igual que ocurre en el caso de las lecciones magistrales. Por su parte, se observa una cierta relajación en el caso de los talleres, pues si bien los dos primeros cursos más del 50\% 
TABLA 5

DISTRIBUCIÓN DE RESULTADOS POR PRUEBAS Y CURSOS

\begin{tabular}{lcccc}
\hline & \multicolumn{2}{c}{ A. MAG ISTRALES } & & \\
& $2008-2009$ & $2009-2010$ & $2010-2011$ & $2011-2012$ \\
\cline { 2 - 5 } Suspenso sin compensación & $2,94 \%$ & $9,62 \%$ & $7,57 \%$ & $15,15 \%$ \\
Suspenso con compensación & $6,47 \%$ & $28,85 \%$ & $5,41 \%$ & $11,52 \%$ \\
Aprobado & $49,41 \%$ & $27,56 \%$ & $42,70 \%$ & $37,58 \%$ \\
Notable & $37,06 \%$ & $31,41 \%$ & $39,46 \%$ & $33,33 \%$ \\
Sobresaliente & $4,12 \%$ & $2,56 \%$ & $4,86 \%$ & $2,42 \%$ \\
\hline & B. LIBRO ABIERTO & & \\
& $2008-2009$ & $2009-2010$ & $2010-2011$ & $2011-2012$ \\
\cline { 2 - 5 } Suspenso sin compensación & $4,73 \%$ & $10,18 \%$ & $4,37 \%$ & $6,33 \%$ \\
Suspenso con compensación & $8,88 \%$ & $10,78 \%$ & $9,84 \%$ & $13,92 \%$ \\
Aprobado & $43,20 \%$ & $34,73 \%$ & $47,54 \%$ & $37,97 \%$ \\
Notable & $37,87 \%$ & $37,72 \%$ & $32,24 \%$ & $36,29 \%$ \\
Sobresaliente & $5,33 \%$ & $6,59 \%$ & $6,01 \%$ & $5,49 \%$ \\
\hline
\end{tabular}

\section{SEMINARIOS (PRUEBAS DE DESARROLLO)}

Suspenso sin compensación

Suspenso con compensación

\begin{tabular}{cccc}
$2008-2009$ & $2009-2010$ & $2010-2011$ & $2011-2012$ \\
\hline $29,93 \%$ & $35,14 \%$ & $45,91 \%$ & $37,32 \%$ \\
$29,93 \%$ & $35,14 \%$ & $26,42 \%$ & $30,62 \%$ \\
$28,57 \%$ & $18,92 \%$ & $18,24 \%$ & $19,62 \%$ \\
$9,52 \%$ & $10,14 \%$ & $6,92 \%$ & $10,53 \%$ \\
$2,04 \%$ & $0,68 \%$ & $2,52 \%$ & $1,91 \%$ \\
\hline
\end{tabular}

Aprobado

Notable

Sobresaliente

\section{SEMINARIOS (PRUEBAS TIPO TEST)}

\begin{tabular}{lcccc} 
& $2008-2009$ & $2009-2010$ & $2010-2011$ & $2011-2012$ \\
\cline { 2 - 5 } Suspenso sin compensación & $2,04 \%$ & $21,62 \%$ & $6,92 \%$ & $15,79 \%$ \\
Suspenso con compensación & $16,33 \%$ & $29,05 \%$ & $35,85 \%$ & $23,92 \%$ \\
Aprobado & $29,25 \%$ & $35,81 \%$ & $37,74 \%$ & $40,67 \%$ \\
Notable & $43,54 \%$ & $12,84 \%$ & $18,87 \%$ & $18,18 \%$ \\
Sobresaliente & $8,84 \%$ & $0,68 \%$ & $0,63 \%$ & $1,44 \%$ \\
\hline \multicolumn{4}{c}{ E. TAL LE RES } \\
Suspenso sin compensación & $2008-2009$ & $2009-2010$ & $2010-2011$ & $2011-2012$ \\
\cline { 2 - 5 } Suspenso con compensación & $2,72 \%$ & $0,68 \%$ & $2,52 \%$ & $2,87 \%$ \\
Aprobado & $10,88 \%$ & $8,11 \%$ & $18,87 \%$ & $4,78 \%$ \\
Notable & $33,33 \%$ & $22,30 \%$ & $44,03 \%$ & $52,15 \%$ \\
Sobresaliente & $52,38 \%$ & $64,19 \%$ & $32,08 \%$ & $39,23 \%$ \\
\hline
\end{tabular}

Fuente: Elaboración propia 
y $60 \%$ alcanzaron la media de notable, en el tercero ese porcentaje se reduce al $30 \%$ para volver a recuperarse parcialmente en el último curso (40\%).

Ahora bien, si nos preguntamos por la satisfacción del alumno con las calificaciones obtenidas, debemos afirmar, como se deduce de la tabla 6, que ésta cambia en función de la actividad. Mientras es elevada en las magistrales, y parcialmente en los talleres, donde las calificaciones son más altas, se reduce mucho cuando hablamos de las obtenidas en las pruebas de los seminarios. Este hecho contrasta con la satisfacción global con el nivel de aprendizaje alcanzado, que pese a la ligera caída que se observa en los dos años centrales, es siempre elevado.

TABLA 6

NIVEL DE SATISFACCIÓN DEL ALUMNO CON LAS CALIFICACIONES OBTENIDAS Y CON EL APRENDIZAJE

\begin{tabular}{|c|c|c|c|c|c|c|c|c|}
\hline & \multicolumn{4}{|c|}{ Magistrales } & \multicolumn{4}{|c|}{ Seminarios } \\
\hline & 2008-2009 & $2009-2010$ & $2010-2011$ & 2011-2012 & 2008-2009 & $2009-2010$ & $2010-2011$ & 2011-2012 \\
\hline Muy satisfecho & $30,2 \%$ & $21,7 \%$ & $15,8 \%$ & $24,8 \%$ & $4,0 \%$ & $3,2 \%$ & $1,3 \%$ & $3,7 \%$ \\
\hline Satisfecho & $55,3 \%$ & $58,0 \%$ & $61,4 \%$ & $59,6 \%$ & $29,6 \%$ & $32,9 \%$ & $19,0 \%$ & $27,5 \%$ \\
\hline Poco satisfecho & $11,2 \%$ & $18,1 \%$ & $19,0 \%$ & $12,4 \%$ & $66,5 \%$ & $63,9 \%$ & $79,7 \%$ & $67,4 \%$ \\
\hline \multirow[t]{3}{*}{ Totalmente insatisfecho } & $3,4 \%$ & $2,2 \%$ & $3,8 \%$ & $3,2 \%$ & $0,0 \%$ & $0,0 \%$ & $0,0 \%$ & $1,4 \%$ \\
\hline & \multicolumn{4}{|c|}{ Talleres } & \multicolumn{4}{|c|}{ Aprendizaje } \\
\hline & $2008-2009$ & 2009-2010 & 2010-2011 & 2011-2012 & 2008-2009 & $2009-2010$ & $2010-2011$ & 2011-2012 \\
\hline Muy satisfecho & $20,7 \%$ & $11,9 \%$ & $4,4 \%$ & $10,1 \%$ & $19,9 \%$ & $20,4 \%$ & $13,5 \%$ & $19,6 \%$ \\
\hline Satisfecho & $46,9 \%$ & $52,5 \%$ & $39,9 \%$ & $46,3 \%$ & $60,8 \%$ & $58,0 \%$ & $63,2 \%$ & $64,8 \%$ \\
\hline Poco satisfecho & $29,1 \%$ & $33,1 \%$ & $44,3 \%$ & $35,8 \%$ & $17,7 \%$ & $20,4 \%$ & $22,1 \%$ & $14,2 \%$ \\
\hline Totalmente insatisfecho & $3,4 \%$ & $2,5 \%$ & $11,4 \%$ & $7,8 \%$ & $1,7 \%$ & $1,2 \%$ & $1,2 \%$ & $1,4 \%$ \\
\hline
\end{tabular}

Fuente: Elaboración propia.

Aunque parece haber un empeoramiento generalizado en la formación del alumno, profundizaremos en los resultados de las pruebas de conocimiento (seminario) para tratar de determinar si esos resultados responden a una peor formación del alumno, o es sólo el resultado de una preferencia del alumno por dirigir su esfuerzo hacia aquellas actividades que les resulta más atractivas de realizar y en las que considera que es más sencillo aprobar, limitándose a alcanzar los mínimos en el caso de las pruebas puramente teóricas.

\subsection{Resultados del bloque de seminarios: pruebas de desarrollo y tipo test}

La actividad de seminarios comporta dos pruebas de evaluación diferentes: las pruebas escritas de desarrollo y la realización de un test al finalizar el semestre sobre el contenido general de la materia.

La comparación en los resultados alcanzados en las pruebas test entre cursos académicos, y entre éstas y las de desarrollo, resulta muy significativa para conocer si ha empeorado o no la formación del alumno, pues durante los cuatro cursos académicos la prueba test ha contado, como ya dijimos, con las mismas preguntas y posibles respuestas a cada una de ellas, habiéndose modificado sólo las preguntas de desarrollo.

En primer término, debemos recordar que los resultados alcanzados por los alumnos en las pruebas tipo test son siempre superiores a los obtenidos en las preguntas de 
desarrollo. No obstante, es necesario profundizar en la relación existente entre las dos pruebas pertenecientes al bloque de seminarios. Así, y como se observa en la figura 2, la relación entre ambas pruebas es positiva en todos los cursos académicos, si bien pueden establecerse diferencias reseñables. En el primer curso académico (2008-2009), las calificaciones del test son en general más elevadas, pues la línea de regresión de ese curso se sitúa por encima de las del resto. Además, para las calificaciones medias de los seminarios (entre el 2 y el 6,5), la línea de regresión tiende a ser bastante plana, no pareciendo existir relación alguna entre la calificación de las pruebas de desarrollo y el test, algo que no sucede en el caso de las calificaciones extremas. Así, en los cursos 2009-2010 y 2010-2011, una baja calificación en las pruebas de desarrollo da lugar a flojos resultados en el test, que no obstante, aumentan más que proporcionalmente al hacerlo la calificación del seminario. Este hecho no se observa, sin embargo, en los otros dos cursos. Por otro lado, cuando se alcanzan altas calificaciones en las pruebas de desarrollo los resultados del test no parecen seguir un patrón claro de comportamiento, y observamos cómo, para una misma calificación en las pruebas de desarrollo, el resultado medio del test cambia mucho de unos cursos a otros.

Aunque en general los resultados del test final son mejores que los de las pruebas de desarrollo, parece que con el tiempo ha aumentado la correlación entre los resultados de ambas actividades, al tiempo que se ha producido un empeoramiento de los mismos.

FIGURA 2

RELACIÓN ENTRE LAS CALIFICACIONES DE LAS PRUEBAS DE DESARROLLO DE LOS SEMINARIOS Y LAS DE TIPO TEST

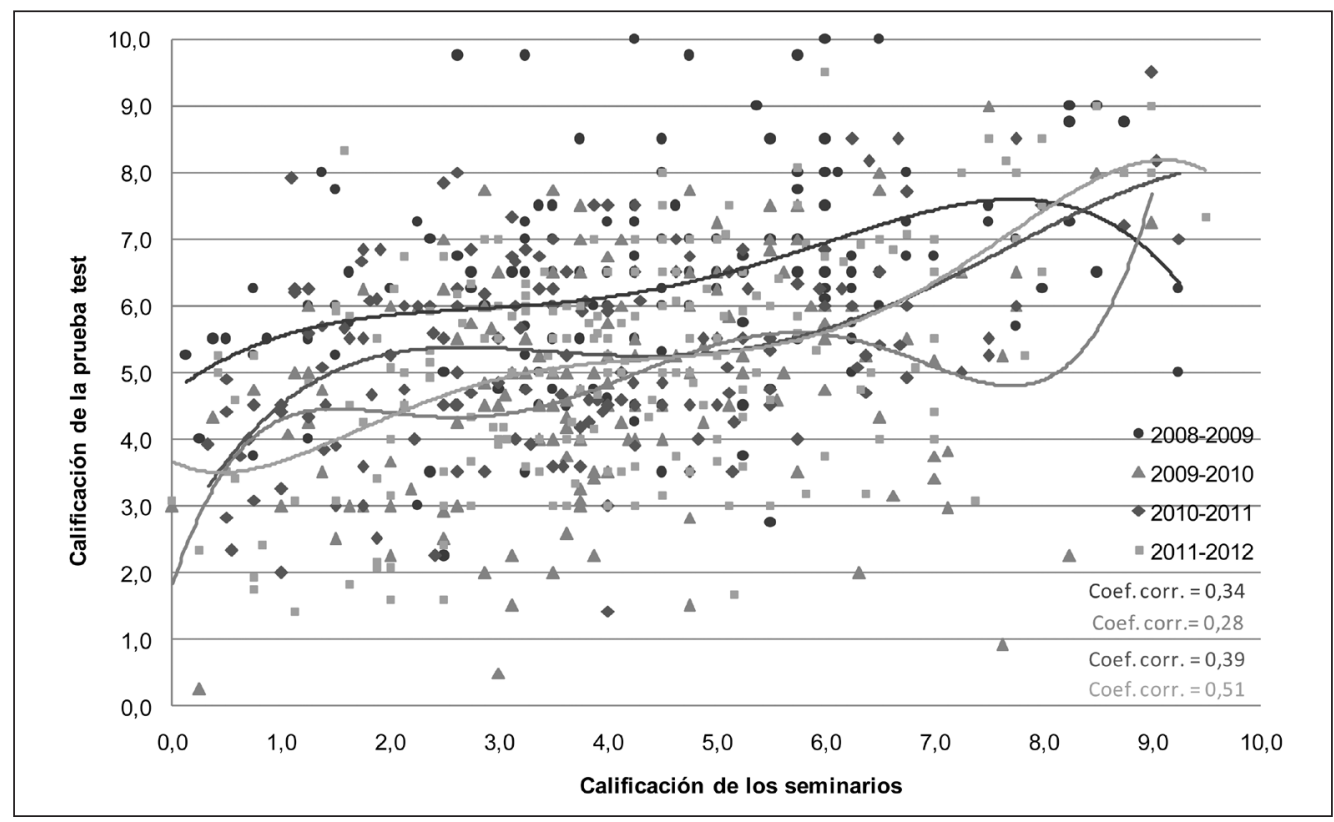

Fuente: Elaboración propia. 


\section{CONCLUSIONES}

Del análisis realizado podemos extraer algunas conclusiones en lo que a la formación del alumno se refiere. Así, hemos observado que el alumno tiende a centrar sus esfuerzos en aquellas actividades que evalúan competencias de habilidad y destrezas, siendo en esas en las que obtiene calificaciones más altas, mientras los resultados de aquellas pruebas que requieren un mayor ejercicio memorístico tienden a ser más bajos. En este sentido, el alumno parece haberse adaptado bastante bien a una metodología interactiva, centrando sus esfuerzos en aquellas actividades que evalúan sus puntos fuertes. Si bien, el hecho de que se observe una cierta relajación en las pruebas de carácter más tradicional nos obliga a preguntarnos si no se estará perjudicando la formación del alumno con una menor adquisición de conocimientos.

Aunque la respuesta a esta pregunta puede dar lugar a opiniones muy controvertidas, es, desde nuestro punto de vista, relativamente sencilla. Así, debemos decir que la formación no se ha visto perjudicada, pues se permite al alumno desarrollar ciertas competencias que resultan esenciales para su incorporación al mercado de trabajo, especialmente en una situación de crisis como la actual, y entre las que se encuentran la planificación y gestión del trabajo, la participación y trabajo en equipos, el desarrollo de las relaciones interpersonales, la toma de decisiones, la comunicación oral y la presentación de ideas, la tolerancia, el respeto y la cooperación.

Además, y aunque los resultados de las pruebas de los seminarios sean peores que las del resto de actividades, no puede concluirse que los conocimientos del alumno se estén deteriorando, pues, por un lado los alumnos afirman estar satisfechos con el nivel de aprendizaje alcanzado aunque lo estén en menor medida con las calificaciones obtenidas, y por otro, también en los talleres, lecciones magistrales y pruebas a libro abierto el alumno adquiere conocimientos y competencias específicas de la materia (véase Rodríguez Esteban y Vieira Aller (2009)). Lo que sí parece claro es la existencia de un sesgo de los estudiantes en favor del trabajo frente al estudio, y de la participación y comprensión de conceptos frente a la actitud más pasiva y de carácter memorístico de los sistemas tradicionales.

Por otro lado, y aunque tanto la metodología, como algunas de las pruebas elaboradas para evaluar la adquisición de competencias se han mantenido invariables de unos cursos a otros, no podemos concluir que los alumnos de ahora, pese a obtener peores resultados que los de los primeros cursos considerados, tengan una mala formación. Sólo podemos atribuir la desigual distribución de los resultados a factores como, el tiempo que dedican al trabajo y al estudio, la mayor o menor disponibilidad de tiempo a medida que otras materias han incorporado metodologías parecidas, e incluso el nivel de exigencia de los profesores, pues la mayoría de los alumnos cubren los mínimos. Así, y como señalan Abalde Paz et al. (2009), las características del alumno y el contexto de la enseñanza, resultan tan esenciales como el propio proceso de enseñanza y aprendizaje en los resultados finales.

En este sentido, la comunicación entre las diferentes unidades docentes para evitar sobrecargas de trabajo, la adaptación a las horas de esfuerzo con las que realmente cuenta la asignatura, y la comunicación permanente con el alumno, pueden ser pasos imprescindibles para mejorar, no sólo los resultados, sino también su formación. 


\section{REFERENCIAS BIBLIOGRÁFICAS}

Abalde Paz, E., Barca Lozano, A., Muñoz Cantero, J. M., \& Ziemer, M. F. (2009). Rendimiento académico y enfoques de aprendizaje: una aproximación a la realidad de la enseñanza superior brasileña en la región norte. Revista de Investigación Educativa, 27 (2), 303-319.

Andreu-Andrés, M. A., \& Labrador-Piquer, M. J. (2011). Formación del profesorado en metodologías de evaluación. Análisis cualitativo. Revista de Investigación en Educación, 9 (2), 236-245.

Brennan, R. L. (2006). Educational measurement. Washington, DC: American Council on Education/Praeger.

Calvo, A., \& Mingorance, A. C. (2010). Evaluación continua de conocimientos vs. de competencias: resultados de la aplicación de dos métodos valorativos diferentes. Revista de Investigación Educativa, 28 (2), 361-384.

Calvo, A., \& Mingorance, A. C. (2011). Valoración universitaria de la formación de competencias: el esfuerzo y la satisfacción de los estudiantes. Educatio siglo XXI, 29 (2), 283-312.

Cano, E. (2008). La evaluación por competencias en la educación superior. Profesorado, Revista de Currículo y Formación del Profesorado, 12, 220-235.

De Jorge, J., Gil, L., Merino, F., \& Sanz, M. (2001). Evidencia empírica de los motivos del absentismo en los estudiantes universitarios. Revista de Investigación en Educación. 9 (2), 76-90.

Fernández, A. (2006). Metodologías activas para la formación de competencias. Educatio siglo XXI, 24, 35-56.

González López, I., \& López Cámara, A. B. (2010). Sentando las bases para la construcción de un modelo de evaluación a las competencias docentes del profesorado universitario. Revista de Investigación Educativa, 28 (2), 403-423.

Inda, M., Álvarez, S., \& Álavarez, R. (2008). Métodos de evaluación en la enseñanza superior. Revista de Investigación Educativa, 26 (2), 539-552.

Ion, G., \& Cano, E. (2011). El proceso de implementación de la evaluación por competencias en la Educación Superior. Un estudio del rol de los cargos académicos. Revista de Investigación en Educación, 9 (2), 246-258.

Joint Committee on Standars for Educational Evaluation (2003). The student evaluation standards. Thousand Oaks, CA: Corwin Press.

Mninisterio de Educación y Ciencia (2006). Directrices para la elaboración de títulos universitarios de Grado y Máster (Documento de trabajo). Madrid: Autor.

Moust, J. H. C., Bouhuijs, P. A. J., \& Schmidt, H. G. (2007). El aprendizaje basado en problemas: guía del estudiante. Cuenca: Ediciones de la Universidad de Castilla-La Mancha.

Múñiz, J., \& Fonseca, E. (2008). Construcción de instrumentos de medida para la evaluación universitaria. Revista de Investigación en Educación, 5, 13-25.

Rodríguez Esteban, A., \& Vieira Aller, M. J. (2009). La formación en competencias en la universidad: un estudio empírico sobre su tipología. Revista de Investigación Educativa, 27 (1), 27-47. 
Segura, M. A. (2009). La evaluación de los aprendizajes basada en el desempeño por competencias. Actualidades Investigativas en Educación, 9 (2), 1-25.

Yáñiz, C. (2006). Planificar la enseñanza universitaria para el desarrollo de competencias. Educatio siglo XXI, 24, 17-34.

Fecha de recepción: 28 de mayo de 2012.

Fecha de revisión: 29 de mayo de 2012.

Fecha de aceptación: 24 de octubre de 2012. 
